\title{
A Conversation with Arshad Desai
}

\author{
INTERVIEWER: LARA SZEWCZAK \\ Scientific Editor, Cell
}

\begin{abstract}
Arshad Desai is a Professor of Cellular and Molecular Medicine at the University of California, San Diego and the Leader of the Laboratory of Chromosome Biology at the Ludwig Institute for Cancer Research.
\end{abstract}

Lara Szewczak: Part of your lab focuses on how kinetochores tie in to the cell cycle. What got you interested in that?

Dr. Desai: One of the most striking things when you watch a movie of mitosis is this incredibly synchronous segregation of the chromosomes where somehow they seem to know exactly when to go. I've always been fascinated by how you coordinate such a complex cellularscale process such that it occurs with remarkable synchrony. Over the years we've appreciated that the kinetochore is the key orchestrator of this process and we now know that one of its mechanisms is by controlling the activity of the main enzyme that drives cells out of mitosis. We recently found that kinetochores are also promoting the activity that drives cells out of mitosis and we believe this may allow a real fine tuning or local regulation of how we control the decision to make sure all the chromosomes are properly lined up, and then you go out of mitosis.

Lara Szewczak: The main enzyme being the APC [anaphase-promoting complex] cyclosome: How do we think about that now?

Dr. Desai: APC/cyclosome [APC/C] was this forbiddingly large complex-16 subunits or so-and we've been struggling to understand how it works. The recent revolution in structural biology has given us a precise picture of its architecture, but also the reasons for its complexity because it has to both drive this reaction of catalysis of specific substrates in a very rapid manner and also be extremely sensitively regulated by the chromosomes so that it doesn't inappropriately get turned on. That's the reason why this is such a complex enzyme. Fundamentally, its main activity is to do ubiquitin ligation on two key substrates, cyclin and securin, and it's the degradation of those substrates that drives cells out of mitosis.

Lara Szewczak: How do the chromosomes talk to the $\mathrm{APC} / \mathrm{C}$ ?

Dr. Desai: We've known for over 20 years that one way that chromosomes talk to the $\mathrm{APC} / \mathrm{C}$ is that the kinetochores, when they're unattached, are generating a "wait anaphase" signal and the point of the signal is to really prevent the APC/C from degrading its substrates. There's a lot of beautiful mechanistic work on that. In particular, the kinetochore is catalyzing a conformational conversion of a key protein called Mad2 [mitotic arrest deficient 2] and this then becomes part of a complex that goes on to inhibit the $\mathrm{APC} / \mathrm{C}$.

Our recent work suggests there's another role of the kinetochore in controlling the $\mathrm{APC} / \mathrm{C}$. What the kinetochore is doing is that it's altering the main enzyme activator of the APC/C known as CDC20 [cell division cycle protein 20] and it's activating CDC20 to turn on the APC/ C. And so there's a balance between positive and negative regulation that's actually anchored on a specific binding site on the kinetochore.

Lara Szewczak: What are the contexts where you'd want the two different functional roles working?

Dr. Desai: If a kinetochore is unattached then the chromosome has not yet found the spindle, and you do not want the cell to go out of mitosis. In that situation, you want to make sure that the kinetochore is stopping the APC/C. If the kinetochore is attached, you also want to make sure the $\mathrm{APC} / \mathrm{C}$ turns on relatively quickly so the cells don't spend an inappropriately long time in mitosis. One way to achieve that is to convert the negative signal into a positive signal. This may also enable local control of APC/C activation, which is something we'd like to explore in the future.

Lara Szewczak: And when you say "local," you mean spatial?

Dr. Desai: Spatially localized control. Yes.

Lara Szewczak: So that you're making this decision for one chromosome microtubule attachment at a time...?

Dr. Desai: Right. You don't want to fully relieve cohesion between paired sisters immediately after attachment, but possibly you may alter locally the cohesin dynamics. This is just speculation at this stage but we know that there is positive and negative regulation going on, so one of our goals is to understand exactly what this is doing in cells.

(C) 2017 Desai. This article is distributed under the terms of the Creative Commons Attribution-NonCommercial License, which permits reuse and redistribution, except for commercial purposes, provided that the original author and source are credited. 
Lara Szewczak: What toggles between the activating and the inhibiting?

Dr. Desai: We believe the toggle is through microtubule attachment to kinetochores. It's been appreciated for a long time that when microtubules attach in a certain configuration the checkpoint signaling reaction is turned off. This would immediately tip the balance toward the activation reaction. A key part of the activation reaction is actually dephosphorylation, so we also suspect that microtubule attachment is delivering the phosphatase to the kinetochore. The mechanism of that is unknown but that would also alter the balance based on attachment.

Lara Szewczak: And that's dephosphorylation of CDC20?

Dr. Desai: Dephosphorylation of CDC20 to go on and bind to the APC/C and then drive cells out of mitosis.

Lara Szewczak: So it's a different way of thinking about CDC20.

Dr. Desai: Actually, our work was inspired by classic biochemical work, which had shown that $\mathrm{CDC} 20$ could be negatively regulated by phosphorylation. The in vivo follow-up on that has not really been there, but I think what we've contributed is to show that it's really controlling the timing of $\mathrm{APC} / \mathrm{C}$ activation, and that the kinetochore is influencing that reaction.

Lara Szewczak: Taking a look beyond figuring out this local mechanism, what do you think are the next big questions for understanding this interplay between getting things attached right and moving the cell on through the cell cycle?

Dr. Desai: There's this very localized interplay between mechanical events where the kinetochore is the major machine that's responsible for coupling to the microtubules and driving the movement of the chromosomes, and these signaling events that are deciding what the cell should do. I think the really big questions are to understand precisely how the mechanics influence the signaling pathways and how they're talking to each other. That's a little challenging because we are not good at integrating forces into studies of signaling mechanisms. That's an area where we still understand relatively little about how the process is working.

I work in the Ludwig Institute for Cancer Research and we know that most solid cancers have aneuploidy or incorrect chromosome numbers and they also have chromosomal rearrangements and the ultimate origin of those is problems with segregation in mitosis. The signaling reactions that are occurring in this very confined space are also relevant to understanding the genesis of that phenotype, which is still quite mysterious in the sense that, unlike DNA repair, we don't have clear links with mutations and the phenotype. There are rare mutations in chromosome segregation machinery in cancer but not at the prevalence that would explain the phenotype that we see in solid cancers. So, we also are very interested in linking this sort of detailed mechanistic understanding to potential dysregulation in cancer.
Lara Szewczak: Have the rare mutations pointed toward any likely suspects?

Dr. Desai: There's mutations in the APC/C that were just recently described. There are also mutations in proteins that are involved in the spindle checkpoint, like Bub1, but their prevalence is extremely low; it's not a high prevalence mutation. Obviously, dysregulation by changes in expression or amplification in this kind of very tightly coordinated pathway could be equally deleterious. That's an area we're interested in because you do see misregulation of a lot of these components in different cancers, but we need to model that in a clean way so we can interpret it and see if there is a link to the genesis of aneuploidy.

Lara Szewczak: Misregulation at the level of gene expression, or at the level of protein stability, or...?

Dr. Desai: Most of the analysis is from genomics so there're both amplifications that are found, and also gene expressions changes. A lot of this is coming out of things like the TCGA [The Cancer Genome Atlas] analysis. We're also curious whether there are germline changes that could explain susceptibility or explain how tumors are different. That's something that we're also exploring.

Lara Szewczak: In a solid tumor, forces can be different because of the stiffness of the cellular matrix. You said we're not good at integrating force with these kinds of studies. What kind of technology is that going to take?

Dr. Desai: We can do force analysis in purified systems with tools like optical traps but it's very challenging to integrate analysis of signaling in there. One exciting area is to use force biosensors in a cellular context. While that's always a bit difficult to interpret, I think that's the best tool we have at this moment to assess potential forces and how they influence reactions.

The other big challenge is that a lot of these reactions involve things with rapid half-lives and posttranslational modifications, so I think that's another challenge as to how we can understand those. It's a golden era for high-resolution cell biology. We have beautiful tools and systems to really try to understand how these complex pathways work but at the same time we are always in the need for new technologies and so we're looking for clever people to come up with things we don't anticipate to try and tackle these questions.

Lara Szewczak: The work we've discussed was largely in worms, but you don't just work on worms. What kinds of questions are you going to take into different systems?

Dr. Desai: We also work with human cells quite a bit, but there our work is much more directed at understanding how the centrosome that organizes the spindle is involved in formation of the spindle and also how it contributes to the process of accurate division. That involves collaborative work where we've developed very clean small molecule inhibitors that allow us to remove centrosomes from cells and then see what happens to division, or the accuracy of chromosome segregation, and also potential therapeutic approaches that derive from that. We're not 
analyzing detailed kinetochore biology in human cells; our colleagues are doing fantastic work on that. We focus much more on trying to get at this question of genesis of cancer cell aneuploidy and cell division-targeted therapies. It's more exploratory and more targeted on centrosomes and spindles.

Lara Szewczak: For the aneuploidy field, what do you think are the most exciting things going on right now?

Dr. Desai: I think the realization that a single lagging chromosome in mitosis can trigger these vast genomic sorts of rearrangements has been a major change in our thinking. The other thing that's really exciting is getting a sense of what aneuploidy is doing to cells. That's a very complex field. One of the struggles we have is that we don't have a good measure of aneuploidy. We can count something with one extra chromosome as aneuploidy or something with 40 extra chromosomes as aneuploidy and we haven't really figured out how to address that challenge and compare that in a clean way to phenotypes. There's been beautiful work done by modeling aneuploidy with single extra chromosomes, but in terms of relating back to the spectrum of changes we see in cancers, that's still been challenging to tackle.

The biggest question I still have personally is, where does it come from? What's the genesis of this? It's clear that once you have these events that then you can actually trigger more aneuploidy and more problems. Is this just chance, or is this actually reflecting some vulnerability that we don't understand yet? This is a big debate in the cancer field, and that's something we would love to contribute to in the future.

Lara Szewczak: So, "chance" versus something that may be "predetermined"... Where are you starting from?

Dr. Desai: We're trying to take cells that are most "wildtype" for us: For human cells, that would be ES [embryonic stem] cells; that's the closest. We are very influenced by a "genetic model" sort of thinking: trying to alter specific classic cancer-related pathways and assess whether there's any obvious induction of aneuploidy phenotypes. The bigger challenge we have is we want to do a quantitative assay that gives us a quick readout. That's something we're trying to work on quite intensively right now. I think that will open up a lot of work. With the advent of single-cell sequencing we can do analyses of genome imbalances but this is still not a very high-throughput approach. We need to be able to score tens of thousands, if not millions, of events to look at the relevant frequencies here. From model organisms, we know the frequency of missegregation is extremely low but we still don't have a very good handle on that in mammals. That's a big question for the aneuploidy field: to get a rigorous baseline measurement.

Lara Szewczak: I want to switch gears completely. Imagine that you're just starting your lab. You have funding and resources, but you can't work on kinetochores. Given the scope of what's going on in science, what would you work on?

Dr. Desai: I do really like the work on microbial communities. I really enjoy that and learning how they function as a unit more than themselves and also how they interact and influence, say, hosts, etc. I'm talking more on the functional side, not so much the genomics side. I think that's a fascinating problem that is getting its due recognition because they are everywhere. When people talk about biomass, it's all microbes, really. There's so much unexplored biology there in terms of the kinds of reactions and the kinds of components and pathways. It's just open terrain in terms of exploration. That area excites me a lot.

One other area that I've actually been stimulated to go into partly by our observations where we found that a lot of the chromosome segregation machinery is doing really unexpected stuff in development, stuff we didn't anticipate. That's also made me think a lot about how things work inside an embryo where you have a lot of cells and a lot of neighbors and you have all these complex morphogenetic events occurring, and how do you achieve that? In C. elegans, we can watch it go from one cell that we just leave in a microscope overnight and there's a larvae the next day, so that's truly remarkable. Trying to take this sort of mechanistic worldview into those systems... that's something I would probably do more.

One other area I think is really exciting is the detailed biophysics of macromolecular machines. This is an area that I have always admired. I have a lot of friends who work in that area and I think you can derive really fundamental principles from those kinds of approaches. That's another area I find really exciting. 


\section{$\$_{\text {CSH\& }}^{\infty}$ Cold Spring Harbor Symposia SYMPOSIA On Quantitative Biology}

\section{A Conversation with Arshad Desai}

Cold Spring Harb Symp Quant Biol 2017 82: 386-388 originally published online April 26, 2018 Access the most recent version at doi:10.1101/sqb.2017.82.034702

Creative This article is distributed under the terms of the

Commons http://creativecommons.org/licenses/by-nc/4.0/, which permits reuse and

License redistribution, except for commercial purposes, provided that the original author and source are credited.

Email Alerting Receive free email alerts when new articles cite this article - sign up in Service the box at the top right corner of the article or click here. 